\title{
Agarol, an ergosterol derivative from Agaricus blazei, induces caspase-independent apoptosis in human cancer cells
}

\author{
TAKAMITSU SHIMIZU ${ }^{1,2}$, JUNYA KAWAI $^{1}$, KENJI OUCHI $^{1}$, HARUHISA KIKUCHI $^{3}$, \\ YOSHITERU OSIMA ${ }^{3}$ and RIKIISHI HIDEMI ${ }^{2}$

\begin{abstract}
${ }^{1}$ Mushroom Research Laboratory, Hokuto Corporation, Nagano 381-0008; ${ }^{2}$ Department of Microbiology and Immunology, Tohoku University Graduate School of Dentistry, Sendai 980-8575; ${ }^{3}$ Laboratory of Natural Product Chemistry, Tohoku University Graduate School of Pharmaceutical Sciences, Sendai 980-8578, Japan
\end{abstract}

Received November 27, 2015; Accepted January 12, 2016

DOI: $10.3892 /$ ijo.2016.3391

\begin{abstract}
Agaricus blazei (A. blazei) is a mushroom with many biological effects and active ingredients. We purified a tumoricidal substance from A. blazei, an ergosterol derivative, and named it 'Agarol'. Cytotoxic effects of Agarol were determined by the MTT assay using A549, MKN45, HSC-3, and HSC-4 human carcinoma cell lines treated with Agarol. Apoptosis was detected by flow cytometry analysis. Reactive oxygen species (ROS) levels and mitochondria membrane potential $(\Delta \Psi \mathrm{m})$ were also determined by flow cytometry. Western blot analysis was used to quantify the expression of apoptosis-related proteins. Agarol predominantly induced apoptosis in two p53-wild cell lines (A549 and MKN45) compared to the other p53-mutant cell lines (HSC-3 and HSC-4). Further mechanistic studies revealed that induction of apoptosis is associated with increased generation of ROS, reduced $\Delta \Psi \mathrm{m}$, release of apoptosis-inducing factor (AIF) from the mitochondria to the cytosol, upregulation of Bax, and downregulation of Bcl-2. Caspase-3 activities did not increase, and z-VAD-fmk, a caspase inhibitor, did not inhibit the Agarol-induced apoptosis. These findings indicate that Agarol induces caspase-independent apoptosis in human carcinoma cells through a mitochondrial pathway. The in vivo anticancer activity of Agarol was confirmed in a xenograft murine model. This study suggests a molecular mechanism by which Agarol
\end{abstract}

Correspondence to: Takamitsu Shimizu, Department of Microbiology and Immunology, Tohoku University Graduate School of Dentistry, 4-1 Seiryo-machi, Aoba-ku, Sendai 980-8575, Japan E-mail: takamitsu.shimizu@dent.tohoku.ac.jp

Abbreviations: A. blazei, Agaricus blazei; AIF, apoptosis-inducing factor; BetA, betulinic acid; $\Delta \Psi \mathrm{m}$, mitochondria membrane potential; PFT- $\alpha$, pifithrin- $\alpha$; ROS, reactive oxygen species; siRNA, small interfering RNA

Key words: Agarol, Agaricus blazei, caspase-independent apoptosis, p53, reactive oxygen species, apoptosis-inducing factor induces apoptosis in human carcinoma cells and indicates the potential use of Agarol as an anticancer agent.

\section{Introduction}

Agaricus blazei (A. blazei), an edible mushroom belonging to the Agaricaceae family, has been traditionally used as a health food supplement for the prevention of cancer, diabetes, hyperlipidemia, arteriosclerosis, and chronic hepatitis (1). In recent years, the mushroom has been used as an immunitystimulating adjuvant in cancer chemotherapy. One of the anticancer substances was postulated to be the $\beta$-glucan fraction (2); orally administered $\beta$-glucan extracted from A. blazei results in tumor regression in tumor-bearing mice (3). Some other compounds of A. blazei, such as ergosterol (lipid fraction) and blazeispirols (skeletal compounds), have antitumor effects due to their anti-angiogenic activities $(4,5)$. In addition, recent studies have found that $\beta$-glucan from $A$. blazei inhibits tumor growth not only by accelerating immune activity but also via direct antitumor activities. An extract from the fruiting bodies of A. blazei has been reported to induce apoptosis in different cancer cell lines, including leukemia, uterine cervical carcinoma, melanoma, and breast adenocarcinoma cells (6). However, the apoptotic mechanism in cancer cells induced by the tumoricidal components from A. blazei is poorly understood. In this study, we show that $A$. blazei extracts induced apoptosis in p53-wild-type cell lines and p53-mutant cell lines. We further purified the tumoricidal substance from A. blazei, an ergosterol derivative, and named it 'Agarol'.

For several decades, apoptosis has been considered to be the principal mechanism of programmed cell death in mammalian cells. Because one of the main objectives of traditional cancer therapy is to enhance cancer cell apoptosis, there have been many studies showing chemotherapeutic agents, including natural compounds, inducing apoptosis by different apoptotic pathways in cancer cells (7). Although caspases were identified as the enzymes that orchestrate apoptotic cell death, it soon became apparent that inhibition of caspase activity may not necessarily preserve cell survival, even with the processes of apoptosis effectively blocked (8). Indeed, numerous reports described that complete caspase inhibition is not able to prevent cell death in vitro or in vivo (9). Interestingly, a study has 
shown that blazeispirol A, isolated from A. blazei, decreases the viability of hepatoma Hep 3B cells, which is not blocked by the pan-caspase inhibitor z-VAD-fmk. Accordingly, this form of cell death has been called caspase-independent cell death to distinguish it from the caspase-dependent apoptotic pathway. In general, many cancer cells have defects in caspase signaling, which allow cancer cells to become resistant to traditional chemotherapy drugs, resulting in a major limitation of cancer treatment. Therefore, the caspase-independent cell death pathway has become an attractive alternative approach for eradicating tumor cells. Mitochondria are central participants in apoptosis and play a direct role in cell death signaling through the well-characterized mitochondrial pathway, which results in the release of apoptogenic factors into the cytosol. Apoptosis-inducing factor (AIF), a mitochondrial protein, induces large-scale DNA fragmentation after nuclear translocation in the caspase-independent apoptotic pathway (10).

Numerous experimental and epidemiological studies have shown that several plant-derived natural products may serve as effective anticancer drugs. Betulinic acid (BetA) has comparatively related structure to ergosterol and belongs to the group of terpenes (11). BetA is found in the bark of white birch trees, and inhibits numerous carcinoma cell lines including lung, colon, liver, pancreatic, breast, ovarian, head and neck, and renal cell lines. p53 is a well-known tumor suppressor that plays a master role in the prevention of tumors by regulating apoptosis. Mutations in p53 have been found in $\leq 50 \%$ of all human cancers and cause an increase in oncogenic phenotypes such as proliferation and tumorigenicity. BetA induces apoptosis independent of wild-type p53 protein, and has a direct effect on mitochondria, resulting in the release of soluble apoptogenic factors (12). Although BetA was reported to have less of a cytotoxic effect on normal cells, BetA induced eryptosis/erythroptosis in human erythrocytes via $\mathrm{Ca}^{2+}$ loading and membrane permeabilization.

In this study, we demonstrated that a novel tumoricidal substance from A. blazei, Agarol, induced apoptosis in cancer cells via differential cytotoxicity based on the p53 status, increased reactive oxygen species (ROS) generation, and decreased mitochondrial membrane potential $(\Delta \Psi \mathrm{m})$, which is involved in the apoptotic pathway. In addition, treatment of cancer cells with Agarol induced only a slight increase in caspase-3 activity, and z-VAD-fmk did not inhibit this Agarolinduced apoptosis in A549 cells. We also observed that AIF plays an important role in Agarol-induced apoptosis signaling in A549 cancer cells. Further understanding of the mechanisms underlying Agarol-induced apoptosis may reveal novel therapeutic avenues for cancer treatment.

\section{Materials and methods}

Isolation of Agarol from A. blazei. The fruiting bodies of A. blaze $i$ were cultivated by the Hokuto Corp. (Nagano, Japan), and $10 \mathrm{~kg}$ was extracted with ethanol at room temperature for 2 days. The ethanol extracts were fractionated by solvent partition between ethyl acetate and water to yield an ethyl acetate soluble fraction. The ethyl acetate extracts $(20.73 \mathrm{~g})$ were applied to a silica gel column, and eluted with n-hexaneethyl acetate-methanol. An active fraction $(250 \mathrm{mg})$, eluted with n-hexane-ethyl acetate (1:3), was further applied to an
ODS column, and eluted with water-acetonitrile-ethyl acetate. The acetonitrile-ethyl acetate (1:1) eluent (24.4 mg) was next applied to a silica gel column, and eluted with chloroformmethanol, resulting in $2.6 \mathrm{mg}$ of Agarol. The structure of the active compound was identified by its ${ }^{1} \mathrm{H}$ and ${ }^{13} \mathrm{C}$ NMR spectra. Its chemical structure is $5 \alpha, 9 \alpha$-epidioxy-(22E)ergosta-7,22-diene-3 $\beta, 6 \beta$-diol, which is comparatively similar to BetA (Fig. 1).

Cell cultures and reagents. HSC-3 and HSC-4 cells (mutanttype p53, human oral squamous cell carcinoma), and A549 cells (wild-type p53, human non-small cell lung adenocarcinoma) were obtained from the RIKEN Cell Bank (Tsukuba, Japan), and MKN45 cells (wild-type p53, human stomach adenocarcinoma) were obtained from the Japanese Cancer Research Resources Bank. HNG-1 (normal human fibroblast cells), derived from gingiva, was isolated. Cells were cultured in RPMI-1640 (Nissui Pharmaceutical, Tokyo, Japan) medium supplemented with $10 \%$ (v/v) heat-inactivated fetal bovine serum, $100 \mathrm{IU} / \mathrm{ml}$ penicillin (Invitrogen, Carlsbad, CA, USA), and $100 \mu \mathrm{g} / \mathrm{ml}$ streptomycin (Invitrogen). Cells were maintained in a humidified atmosphere of $95 \%$ air and $5 \% \mathrm{CO}_{2}$ at $37^{\circ} \mathrm{C}$. Pan-caspase inhibitor z-VAD-fmk (MBL, Nagoya, Japan), p53 inhibitor pifithrin- $\alpha$ (PFT- $\alpha$ ) (Wako Pure Chemical Industries, Ltd., Osaka, Japan), and free radical scavenger N-acetyl-L-cysteine (NAC) (Calbiochem, La Jolla, CA, USA) were purchased. Betulinic acid (Enzo Life Science, NY, USA) was provided as pure substance and dissolved in dimethyl sulfoxide. All inhibitors used in this study were incubated with cells for $1 \mathrm{~h}$, followed by each indicated treatment without washing.

Cytotoxicity assays. The cytotoxic effects of Agarol were evaluated using an MTT (WST-8) colorimetric assay kit (Dojindo, Kumamoto, Japan). Apoptotic cells were assayed by the TUNEL method using the Mebstain apoptosis kit direct (MBL) for flow cytometric analysis (FACSCalibur; BectonDickinson, San Jose, CA, USA).

Caspase-3 assay. Caspase-3 activity was measured using a CPP32/caspase-3 colorimetric protease assay kit (MBL). Absorbance of extracts from cells treated with Agarol was measured at $405 \mathrm{~nm}$ in a microplate reader.

Western blotting. SDS-PAGE and western blots for whole or nuclear proteins were performed according to standard procedures. Immunodetection was performed with HRP-conjugated secondary antibodies and visualized with the chemiluminescence detection method. Primary antibodies were anti-Bcl-2 (BD Biosciences, San Jose, CA, USA), Bax (Cell Signaling Technology, Inc., Danvers, MA, USA), PARP (Cell Signaling Technology), AIF (Cell Signaling), $\beta$-actin (Funakoshi, Tokyo, Japan), and Lamin A (Biolegend, San Diego, CA, USA). Secondary antibodies were HRP-conjugated sheep anti-mouse IgG (GE Healthcare, Piscataway, NJ, USA) and goat anti-rabbit IgG (Santa Cruz Biotechnology, Santa Cruz, CA, USA).

siRNA transfection. Cells were transiently transfected with either $40 \mathrm{nM}$ negative control small interfering (si)RNA (Bioneer, Deajeon, Korea) or 10 nM AIF siRNA (Santa Cruz 

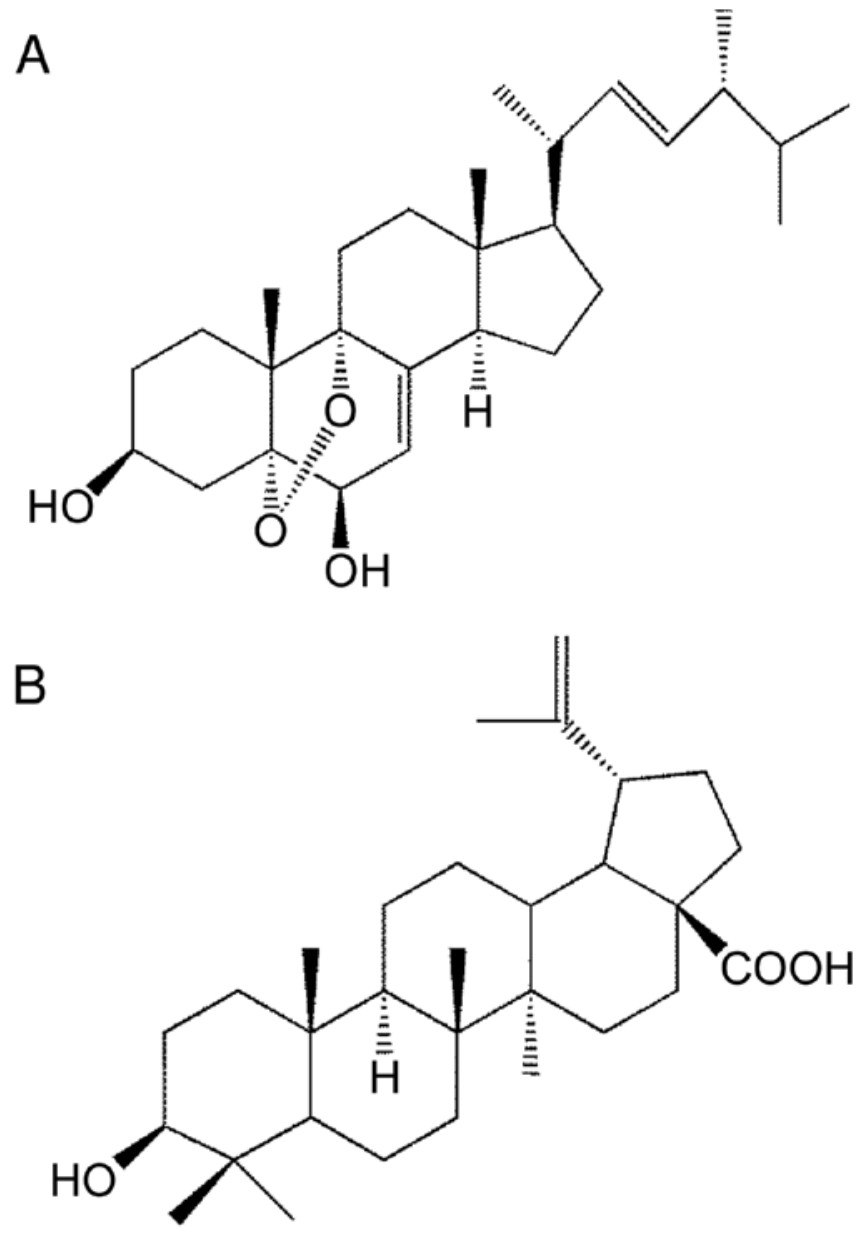

Figure 1. Chemical structures of Agarol (A) and BetA (B).

Biotechnology) at $80 \%$ confluence using Lipofectamine 2000 Transfection Reagent (Invitrogen).

Measurement of ROS and $\triangle \Psi m$. Intercellular ROS production was measured using ROS detection reagents (Invitrogen). Cells were treated with Agarol for $4 \mathrm{~h}$. After incubation, cells were exposed to Carboxy- $\mathrm{H}_{2}$ DCFDA (C400) for $30 \mathrm{~min}$ at $37^{\circ} \mathrm{C}$, harvested, and then analyzed using FACSCalibur. Similarly, $\Delta \Psi \mathrm{m}$ was measured using the JC-1 Mitochondrial Membrane Potential Assay kit (Cayman Chemical Co., MI, USA).

In vivo tumor study of Agarol. Male C57BL/6 mice, 7-weekold mice, were used to confirm the toxicity of Agarol. A series of Agarol doses in PBS were tested. Mice were injected i.p. with 10 or $100 \mathrm{mg} / \mathrm{kg} /$ day of Agarol every 3 days for 3 weeks. The drug-free PBS were administered as vehicle controls. Mice were monitored and weighed every 3 days during the course of 3 weeks. Toxicity was assessed as a percent of weight loss. The values presented are the mean $\pm \mathrm{SE}(\mathrm{n}=3$ in each group). The animal protocol was approved by the Institute for Animal Experimentation, Tohoku University Graduate School of Medicine (no. 2014shidou-018).

Female severe combined immunodeficiency (SCID) mice, 5-week-old mice, were used to in vivo tumor modeling study of Agarol. A549 cells (1 $1 \times 10^{7}$ cells/mouse) suspended in PBS were injected subcutaneously into the right flank of the mice. After
2 weeks, when the size of solid tumor in tumor-bearing SCID mice reached $150-200 \mathrm{~mm}^{3}$, the tumor-bearing SCID mice were treated with Agarol or BetA via i.p. administration at the dosage of $0,10,30$ and $100 \mathrm{mg} / \mathrm{kg} /$ day with Agarol or $100 \mathrm{mg} / \mathrm{kg} / \mathrm{day}$ with BetA every 3 days for 3 weeks. After 3 weeks, the tumors were resected and measured. The values presented are the mean $\pm \mathrm{SE}$ ( $\mathrm{n}=10$ in each group). The animal protocol was approved by the Ethics Committee of KAC Corporation (no. 14-0921).

In situ detection of apoptosis by the TUNEL assay. Xenograft tumors were resected and fixed in 10\% formalin neutral buffer solution (Wako Pure Chemical Industries), and embedded in paraffin and 5-micron sections. Apoptotic tumor cells were determined by the TUNEL method using the Apoptotic In Situ Detection kit Wako (Wako Pure Chemical Industries) according to the manufacturer's instructions.

Statistical analysis. Data are given as the mean \pm SE. When required, multiple comparisons were made by Scheffe's test. P-values $<0.05$ were considered as statistically significant.

\section{Results}

Cytotoxic effects of Agarol in human cancer cell lines. The structure of our novel ergosterol derivative, Agarol, is shown in Fig. 1A, and the chemical structure of plant-derived BetA, which has been shown to induce cell death in various cancer cell lines, is shown in Fig. 1B. We studied the cytotoxic effects of Agarol in comparison with BetA for anticancer activity of both compounds. We first examined human cancer cell lines with either wild-type or mutant p53 for their sensitivity to Agarol using MTT cytotoxicity assay. Each cell line was treated with Agarol $(0-3 \mu \mathrm{g} / \mathrm{ml})$ for $24 \mathrm{~h}$ (Fig. 2A and B). Agarol treatment decreased the viability of cells with wildtype p53 (A549 and MKN45) in a dose-dependent analysis of cell viability, while higher overall decrease of cell viability was observed with high doses of Agarol in the cell lines with mutant p53 (HSC-3 and HSC-4). $\mathrm{IC}_{50}$ values $(24 \mathrm{~h})$ were $\sim 0.26 \mu \mathrm{g} / \mathrm{ml}$ (A549), $0.34 \mu \mathrm{g} / \mathrm{ml}$ (MKN45), $1.72 \mu \mathrm{g} / \mathrm{ml}$ (HSC3), and $1.94 \mu \mathrm{g} / \mathrm{ml}$ (HSC-4). Exposure of BetA (0-10 $\mu \mathrm{g} / \mathrm{ml})$ to A549 cells also triggered cell death, as indicated by a dosedependent analysis of cell viability (Fig. 2C). In addition, to detect the growth inhibition of Agarol $(1 \mu \mathrm{g} / \mathrm{ml})$ - and BetA $(10 \mu \mathrm{g} / \mathrm{ml})$-exposed A549 cells, the cells were treated for various times (0-48 h). Agarol showed more potent suppressive effect than BetA (Fig. 2D). Cell viability of normal fibroblast cells (HNG-1) was not reduced by Agarol, similar to BetA-treated cells (Fig. 2E).

Apoptosis induced by Agarol in A549 cells. To determine whether the Agarol-induced cell death of A549 cells showed apoptotic characteristics, we analyzed the induction of nucleosome fragmentation by TUNEL staining. As shown in Fig. 3A and $\mathrm{B}$, Agarol $(1 \mu \mathrm{g} / \mathrm{ml})$ treatment for $24 \mathrm{~h}$ induced apoptosis in A549 cells. In addition, we investigated possible pathways responsible for the apoptotic effects of Agarol, and examined the activation of caspase-3 in Agarol- or BetA-treated A549 cells. Agarol induction was undetectable in caspase-3 activity (Fig. 3C); and, a pan-caspase inhibitor, z-VAD-fmk, did not inhibit the Agarol-induced apoptosis in treated cancer cells 

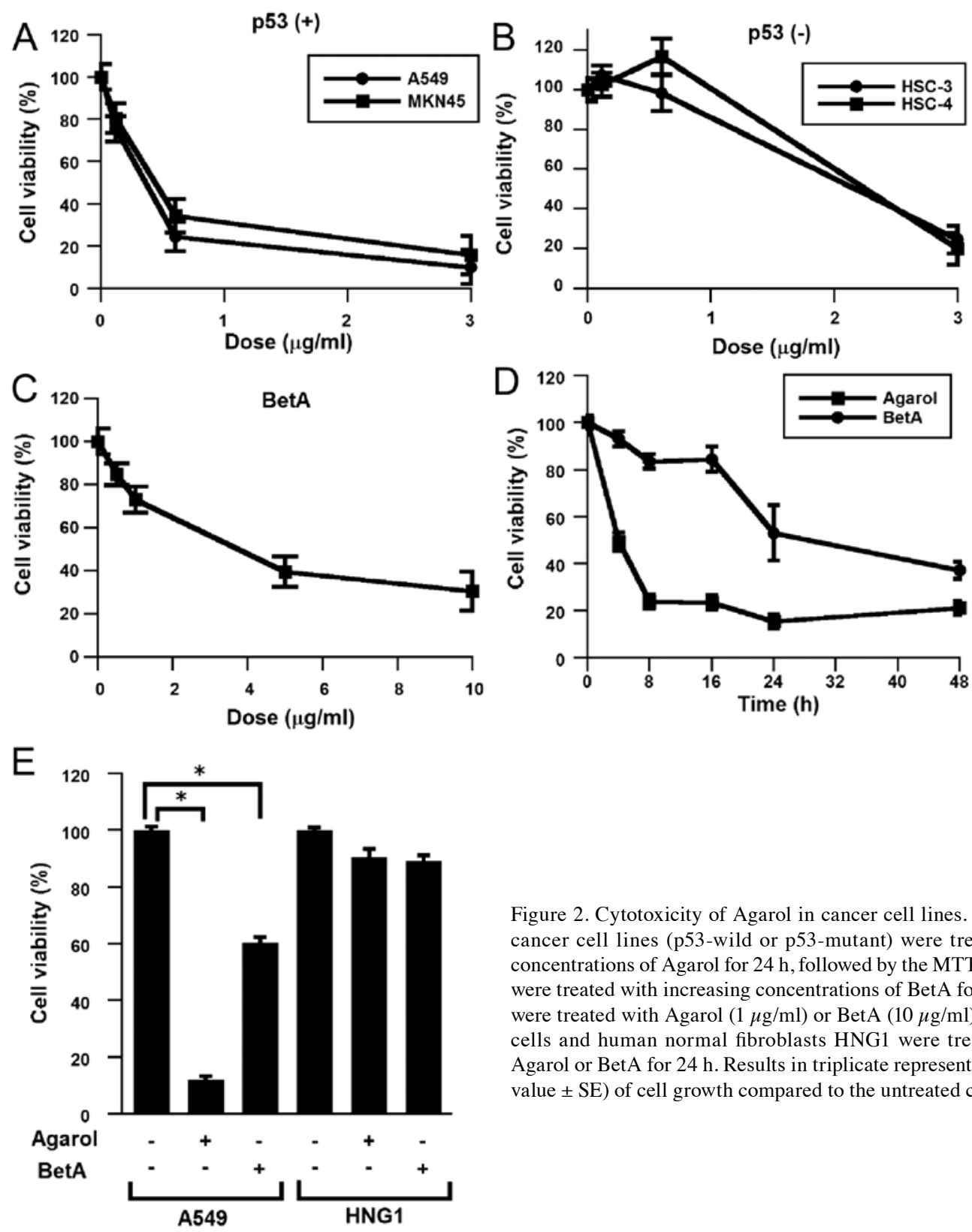

Figure 2. Cytotoxicity of Agarol in cancer cell lines. (A and B) Respective cancer cell lines (p53-wild or p53-mutant) were treated with increasing concentrations of Agarol for $24 \mathrm{~h}$, followed by the MTT assay. (C) A549 cells were treated with increasing concentrations of BetA for $24 \mathrm{~h}$. (D) A549 cells were treated with Agarol $(1 \mu \mathrm{g} / \mathrm{ml})$ or BetA $(10 \mu \mathrm{g} / \mathrm{ml})$ up to $48 \mathrm{~h}$. (E) A549 cells and human normal fibroblasts HNG1 were treated with $2 \mu \mathrm{g} / \mathrm{ml}$ of Agarol or BetA for $24 \mathrm{~h}$. Results in triplicate represent the percentage (mean value $\pm \mathrm{SE}$ ) of cell growth compared to the untreated control. ${ }^{*} \mathrm{P}<0.05$.

(Fig. 3D). In contrast, BetA induced a significant increase in caspase- 3 activity for 24 and 48 h in A549 cells, which was clearly inhibited by z-VAD-fmk. These data indicate that Agarol induced caspase-independent apoptosis in A549 cells.

Mitochondrial dysfunction induced by Agarol in A549 cells. Mitochondrial dysfunction, including the loss of $\Delta \Psi \mathrm{m}$, permeability transition, and release of apoptosis-related factors from the mitochondria into the cytosol or nucleus, is frequently associated with chemotherapy-induced apoptosis. Because BetA is thought to have a direct effect on the mitochondria, we treated A549 cells with Agarol for $4 \mathrm{~h}$ and observed a significant change of $\Delta \Psi \mathrm{m}$. JC-1 staining revealed significant loss of mitochondrial $\Delta \Psi \mathrm{m}$ in the treated group (Fig. 3E). Bcl-2 and Bax have been shown to be important regulators in the mitochondrial apoptotic pathway. Next, the effect of Agarol on expression of Bcl-2 family proteins was investigated in A549 and HSC-3 cells. The cytosol of Agarol-treated cells was

collected at the indicated time-points, and expression of Bcl-2 and Bax proteins was determined by western blot analysis. As shown in Fig. 3F, the level of Bcl-2 decreased after treatment, while Agarol treatment increased the protein expression of Bax in a time-dependent manner. In addition, treatment with Agarol resulted in a time-dependent generation of cleaved PARP in A549 cells.

Agarol-induced apoptosis through AIF-dependent pathways. Mitochondria damage results in the release of pro-apoptotic protein (for example, AIF), which triggers caspase-independent cell death. To determine whether Agarol induces the translocation of AIF from the mitochondria to the nucleus in A549 cells, cells were treated with $1 \mu \mathrm{g} / \mathrm{ml}$ of Agarol for $8 \mathrm{~h}$ and analyzed for AIF protein expression in nuclear fractions. Enhanced nuclear translocation of AIF was evident after treatment (Fig. 4A). In A549 and HSC-3 cells transfected with AIF-specific siRNA, knockdown of the AIF gene effectively 

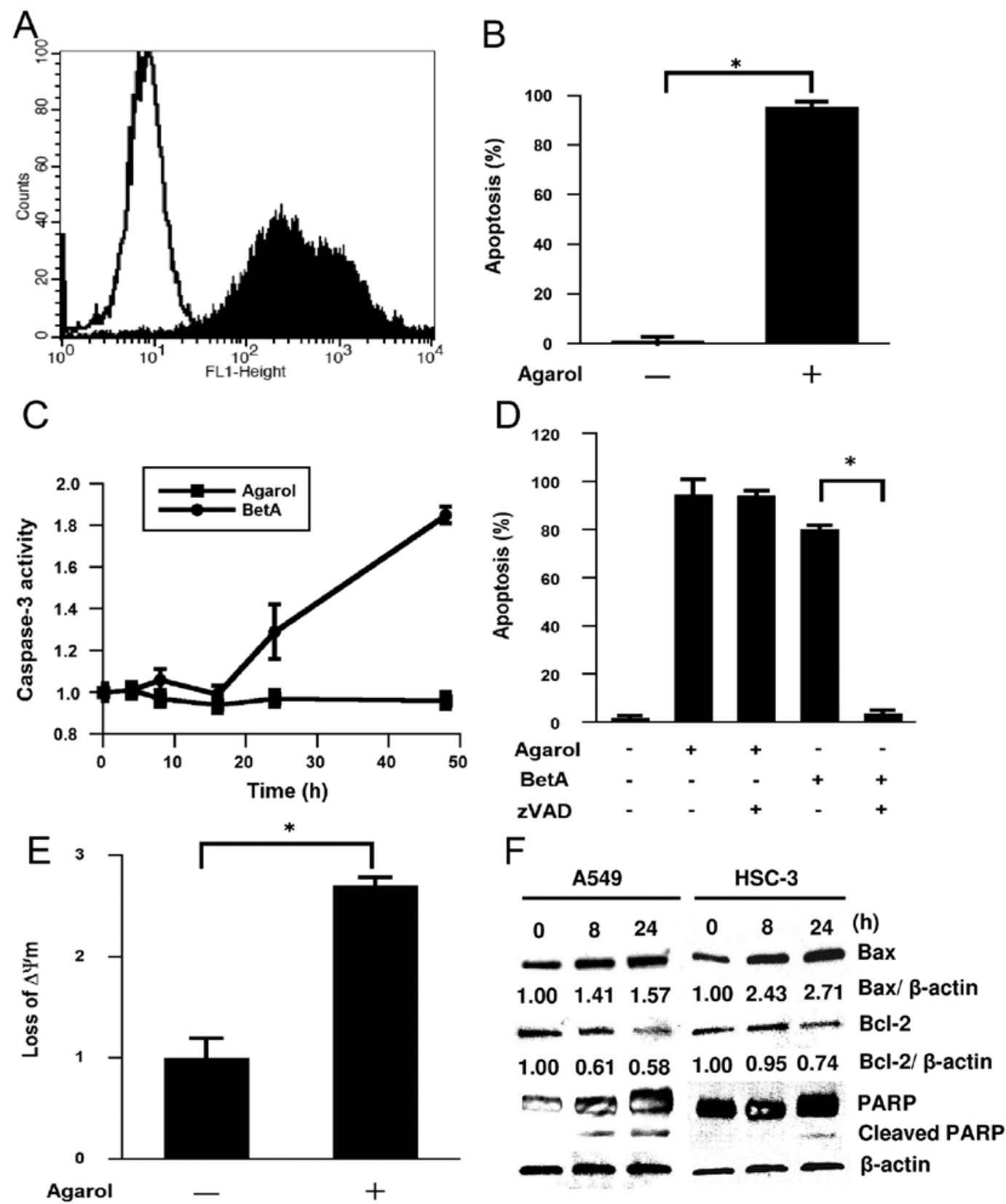

Figure 3. Induction of apoptosis by Agarol in A549 cells. (A) Cells were treated with Agarol ( $1 \mu \mathrm{g} / \mathrm{ml})$ for $24 \mathrm{~h}$, stained with the TUNEL method and evaluated by cytometric analysis. Flow cytometric histograms depict apoptotic cells in untreated control (unshaded) or Agarol-treated (shaded) cells. (B) The percentage of apoptotic cells in triplicate was expressed (mean value \pm SE) in each group. (C) Cells were treated with Agarol $(1 \mu \mathrm{g} / \mathrm{ml})$ or BetA (10 $\mu \mathrm{g} / \mathrm{ml})$ for $48 \mathrm{~h}$, and cytosolic cell lysates were prepared at the indicated times and assayed for DEVDase activity. Results in triplicate represent the ratio (mean value \pm SE) to the untreated control. (D) Cells were pretreated with z-VAD-fmk $(20 \mu \mathrm{M})$ for $1 \mathrm{~h}$ and then treated with Agarol or BetA for $24 \mathrm{~h}$. The percentage of apoptotic cells was determined by TUNEL staining. (E) Cells were treated with Agarol for $4 \mathrm{~h}$, incubated with JC-1 for $20 \mathrm{~min}$, and then subjected to flow cytometric analysis. Results in triplicate represent the ratio (mean value \pm SE) to the untreated control. (F) A549 cells and HSC-3 cells were treated with Agarol (A549 cells, $1 \mu \mathrm{g} / \mathrm{ml}$, HSC-3 cells, $3 \mu \mathrm{g} / \mathrm{ml}$ ) for 0-24 h, and then the cell lysates were harvested. Equal amounts of protein from each sample were loaded for western blotting for determination of Bcl-2, Bax and PARP protein expression. After immunoblotting, the film was scanned and the magnitude of signals were quantified. $\beta$-actin was used as a loading control. ${ }^{*} \mathrm{P}<0.05$.

reduced the cellular level of AIF protein (Fig.4B). Furthermore, siRNA transfection significantly reduced Agarol-induced apoptosis in cells (Fig. 4C), suggesting that Agarol-induced caspase-independent apoptosis is a result of the release of AIF from the mitochondria to the nucleus in A549 and HSC-3 cells.

Involvement of Agarol-induced ROS generation in the p53 pathway. We found that wild-type p53 cells were highly sensi- tive to apoptosis induced by Agarol (Fig. 2A). We examined the effect of pifithrin- $\alpha$ on Agarol-treated wild-type (A549 and MKN45) and mutant p53 cell lines (HSC-3) to confirm the possible role of p53 in Agarol-induced apoptosis. Exposure to pifithrin- $\alpha$ (PFT- $\alpha, 50 \mu \mathrm{M})$ for $8 \mathrm{~h}$ clearly reduced Agarolinduced apoptosis in wild-type p53 cells (A549 and MKN45) (Fig. 5A). In BetA-treated A549 cells a significant effect of PFT- $\alpha$ was not observed (Fig. 5B). 
A

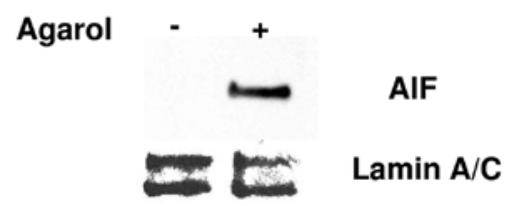

B

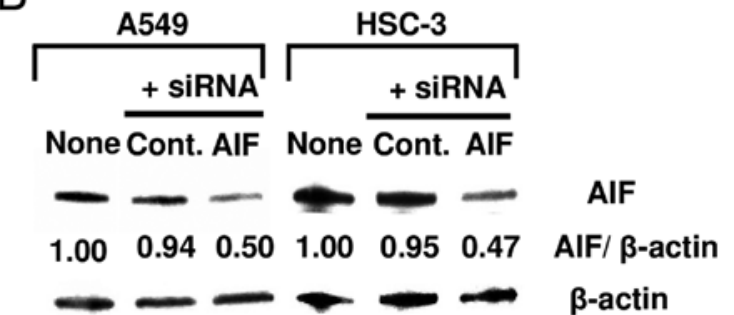

C

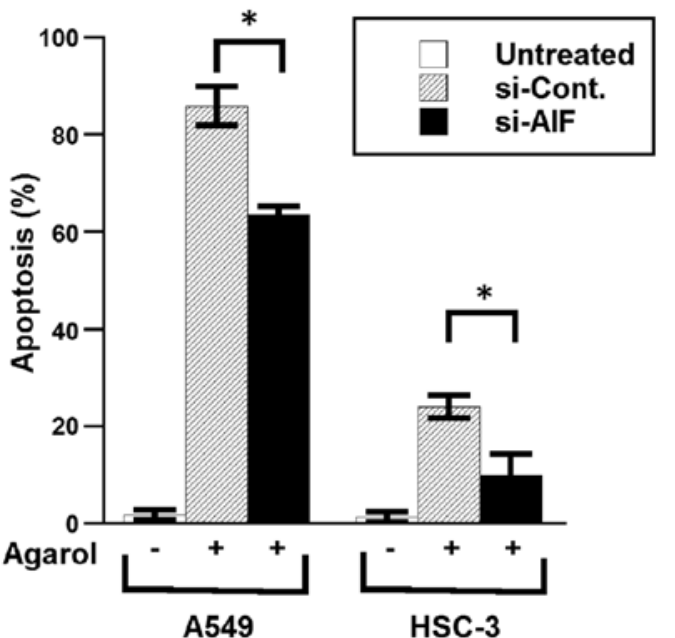

Figure 4. AIF-dependent Agarol-induced apoptosis in A549 cells. (A) A549 cells were treated with Agarol $(1 \mu \mathrm{g} / \mathrm{ml})$ for $8 \mathrm{~h}$. Nuclear proteins were extracted for determination of AIF expression, and equal amounts of nuclear fraction were loaded for western blotting. Lamin $\mathrm{A} / \mathrm{C}$ was used as a loading control. (B) A549 and HSC-3 cells transfected with AIF siRNA were incubated for $24 \mathrm{~h}$. Nuclear proteins were extracted for determination of AIF expression. (C) Cells transfected with AIF siRNA or control siRNA were treated with Agarol (A549 cells, $1 \mu \mathrm{g} / \mathrm{ml}$; HSC-3 cells, $3 \mu \mathrm{g} / \mathrm{ml}$ ) for $24 \mathrm{~h}$. The percentage of apoptotic cells was determined by TUNEL staining. Results in triplicate represent the percentage (mean value $\pm \mathrm{SE}$ ) of apoptotic cells. ${ }^{*} \mathrm{P}<0.05$.
We investigated whether Agarol-induced apoptosis is involved in ROS generation in A549 cells. Cells were examined for evidence of oxidative stress using a peroxide-DCF fluorescence assay. ROS accumulation was observed at 4 and $8 \mathrm{~h}$ of treatment with Agarol in A549 cells (Fig. 6A). In A549 and HSC- 3 cells pretreated for $1 \mathrm{~h}$ with $5 \mathrm{mM}$ NAC, a ROS scavenger, followed by Agarol treatment, NAC reduced Agarol-induced apoptosis in A549 cells, but not in HSC-3 cells (Fig. 6B). Because p53 influences mitochondrial ROS generation, we investigated whether the Agarol-induced ROS generation was associated with the p53 pathway in A549 cells by pretreating cells for $1 \mathrm{~h}$ with PFT- $\alpha$, and observed that the Agarol-induced ROS generation was significantly decreased in A549 cells, but not in HSC-3 cells (Fig. 6C). These results indicated that ROS generation is involved in Agarol-induced apoptosis, and the p53 pathway is involved upstream of this activity in A549 cells.

Anticancer effects of Agarol in a mouse xenograft model with A549. When Agarol was tested for in vivo activity of C57BL/6 mice, the top dosage employed of $100 \mathrm{mg} / \mathrm{kg} /$ day every 3 days i.p. administration for 3 weeks exhibited only weak toxicity at reducing body weight (Fig. 7A). To evaluate the mono-therapeutic efficacy of Agarol against human cancer in vivo, a SCID model was adopted. Fig. 7B demonstrates that single-agent Agarol substantially inhibited A549 human lung adenocarcinoma growth as compared with vehicle control mice $(\mathrm{P}<0.05)$. The treatment with Agarol delayed the growth of the cancer in xenograft mice, but did not cause complete remissions. As seen in Fig. 7C, treatment with Agarol (30 mg/kg/day) or BetA $(100 \mathrm{mg} / \mathrm{kg} / \mathrm{day})$ every 3 days for 3 weeks, showed apoptotic features (arrow) in tumor-bearing SCID mice.

\section{Discussion}

The well-known component of A. blazei, $\beta$-glucan ( $\beta 1-3, \beta 1-6$ linked), inhibits the growth of sarcoma in mice and improves the quality of life via elevating immune activity in cancer patients (13). In addition, the polysaccharide-protein complex
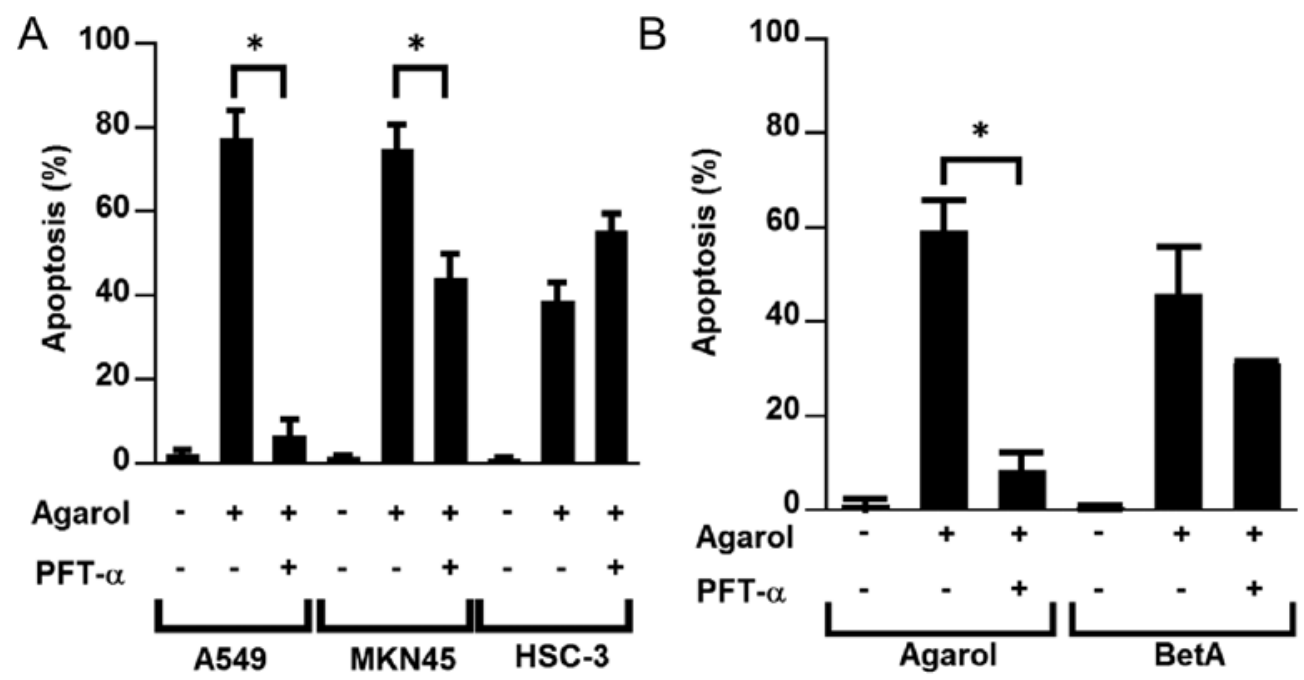

Figure 5. p53-dependent apoptosis by Agarol. (A) Each cell line pretreated for $1 \mathrm{~h}$ with pifithrin- $\alpha$ (PFT- $\alpha, 50 \mu \mathrm{M})$, were treated for $24 \mathrm{~h}$ with $1 \mu \mathrm{g} / \mathrm{ml}$ of Agarol. The percentage of apoptotic cells was determined by TUNEL staining. (B) A549 cells pretreated with PFT- $\alpha$ were treated with Agarol (1 $\mu \mathrm{g} / \mathrm{ml})$ or BetA $(10 \mu \mathrm{g} / \mathrm{ml})$. Results in triplicate represent the percentage (mean value $\pm \mathrm{SE}$ ) of apoptotic cells. ${ }^{*} \mathrm{P}<0.05$. 

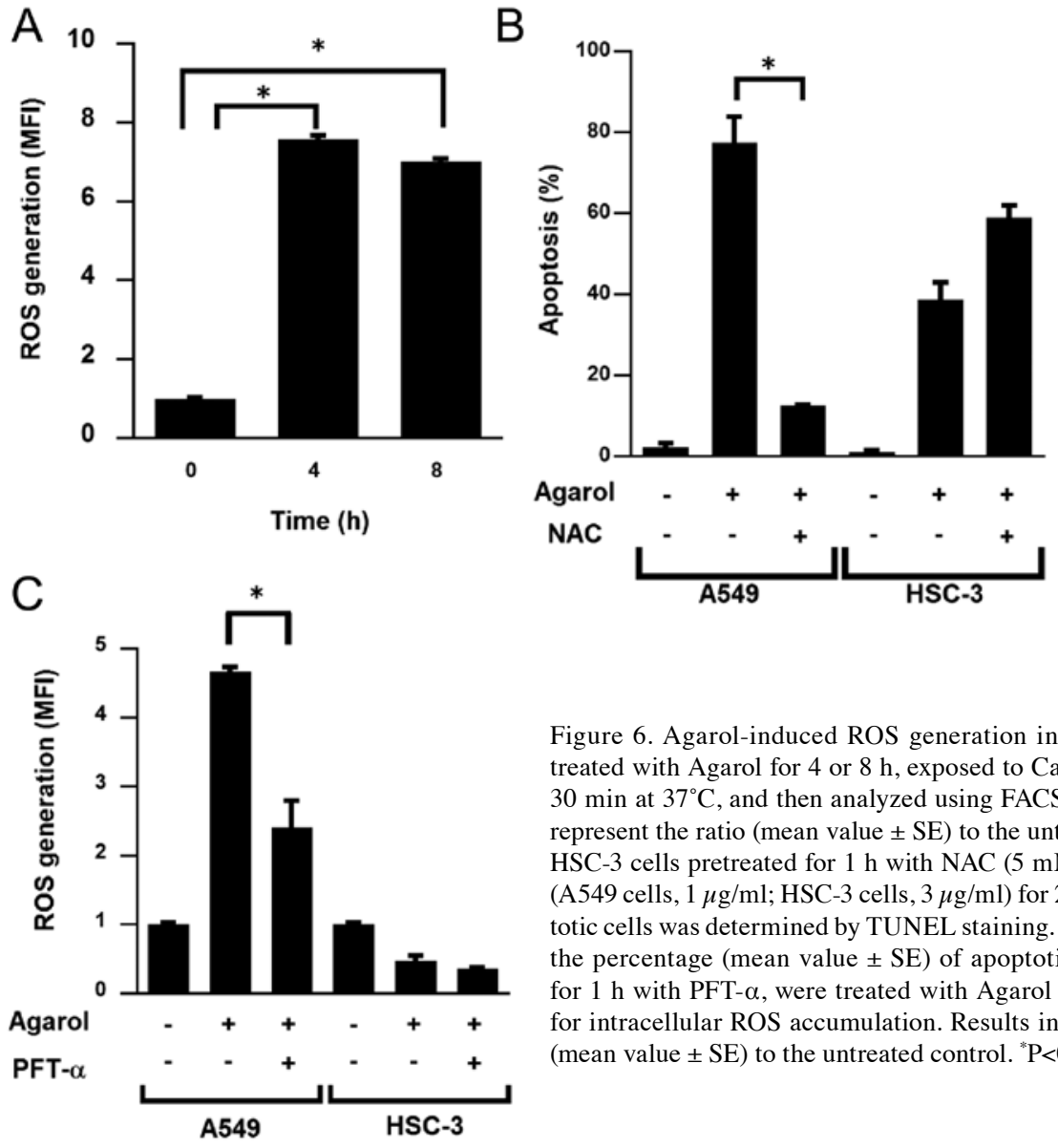

Figure 6. Agarol-induced ROS generation in A549 cells. (A) Cells were treated with Agarol for 4 or $8 \mathrm{~h}$, exposed to Carboxy- $\mathrm{H}_{2}$ DCFDA (C400) for $30 \mathrm{~min}$ at $37^{\circ} \mathrm{C}$, and then analyzed using FACSCalibur. Results in triplicate represent the ratio (mean value $\pm \mathrm{SE}$ ) to the untreated control. (B) A549 and HSC-3 cells pretreated for $1 \mathrm{~h}$ with NAC (5 mM), were treated with Agarol (A549 cells, $1 \mu \mathrm{g} / \mathrm{ml}$; HSC-3 cells, $3 \mu \mathrm{g} / \mathrm{ml}$ ) for $24 \mathrm{~h}$. The percentage of apoptotic cells was determined by TUNEL staining. Results in triplicate represent the percentage (mean value \pm SE) of apoptotic cells. (C) Cells pretreated for $1 \mathrm{~h}$ with PFT- $\alpha$, were treated with Agarol for $4 \mathrm{~h}$. Cells were analyzed for intracellular ROS accumulation. Results in triplicate represent the ratio (mean value $\pm \mathrm{SE}$ ) to the untreated control. ${ }^{*} \mathrm{P}<0.05$.

also shows antitumor activities due to immunological hostmediated mechanisms (14). There is some evidence that the $\beta$-glucan from A. blazei inhibits tumor growth through its direct antitumor activity against aberrantly activated signaling pathways in cancer cells (15). Most studies concerning the mechanisms of $\beta$-glucans have demonstrated that the major antitumor activity of A. blazei extracts may be the induction of apoptosis and cell cycle arrest. Treatment with $\beta$-D-glucan extracted from A.blazei directly stimulates apoptotic signaling in HRA ovarian cancer cells (16). Proteoglycans from A. blazei induce cell cycle arrest and apoptosis in gastric cancer cells and leukemia U937 cells (17). Among other compounds found in A. blazei, it was demonstrated that blazeispirol A, which is the active anti-hepatoma compound in an ethanolic extract of an A. blazei fermentation product, decreases the viability of hepatoma Hep 3B cells by inducing both caspase-dependent and caspase-independent cell death (5). The pro-vitamin D2, ergosterol, is abundant in mushrooms and has been shown to have chemoprevention activity. Ergosterol peroxide attenuates the growth of prostate cells triggering an apoptotic process. However, detailed research of the antitumor components isolated using different extraction and purification methods is lacking. In this study, we used a novel naturally-occurring compound, Agarol, isolated from an extract of A. blazei.

In this study, Agarol at concentrations of $3 \mu \mathrm{g} / \mathrm{ml}$ and above significantly inhibited the growth and viability of human tumor cells with different genetic backgrounds and status of p53. The sensitivity of these cells to Agarol is variable; A549 and MKN45 cells are sensitive to Agarol, whereas HSC-3 and

HSC-4 cells are moderately resistant (as shown by MTT cell viability assays in Fig. 2A and B). The presence of wild-type p53 in cancer cells facilitated apoptotic cell death in response to Agarol treatment as determined by TUNEL staining, whereas cancer cells with a mutated form of p53 appeared to some degree resistant to Agarol-induced apoptosis. In contrast to Agarol, it has been shown that BetA mediated tumor cell death in 3 different adherent human melanomas, irrespective of p53 status (18). p53 regulates the transcription of genes that play an important role in apoptosis. Apoptosis can be induced by p53 through several pathways, one of which involves the Bcl-2 family (19). Bax protein is a p53 target and promotes the release of cytochrome $c$ from the mitochondria. On the other hand, anti-apoptotic proteins, such as Bcl-2, are transcriptionally suppressed by $\mathrm{p} 53$, and suppress the release of cytochrome $\mathrm{c}$ that activates the effectors of apoptosis. The ethanolic extracts of A. blazei, which are enriched in blazeispirols (A and C), upregulated expression of pro-apoptotic Bax protein and downregulated expression of anti-apoptotic Bcl-2 protein in human hepatocellular carcinoma cell lines (20). It was shown that blazeispirol A $(4 \mu \mathrm{g} / \mathrm{ml})$ decreased Bcl-2 and $\mathrm{Bcl}-\mathrm{xL}$ expression and increased Bax expression in a timedependent manner (5). Agarol-induced apoptosis may be, at least in part, regulated by p53 function, via apoptotic promotion by Bax and Bcl-2.

Some reports suggest a role for ROS as a potential mediator of p53-dependent apoptosis. For example, excessive calcium has been reported to induce permeabilization of the mitochondrial outer membrane and, in turn, increase the release 

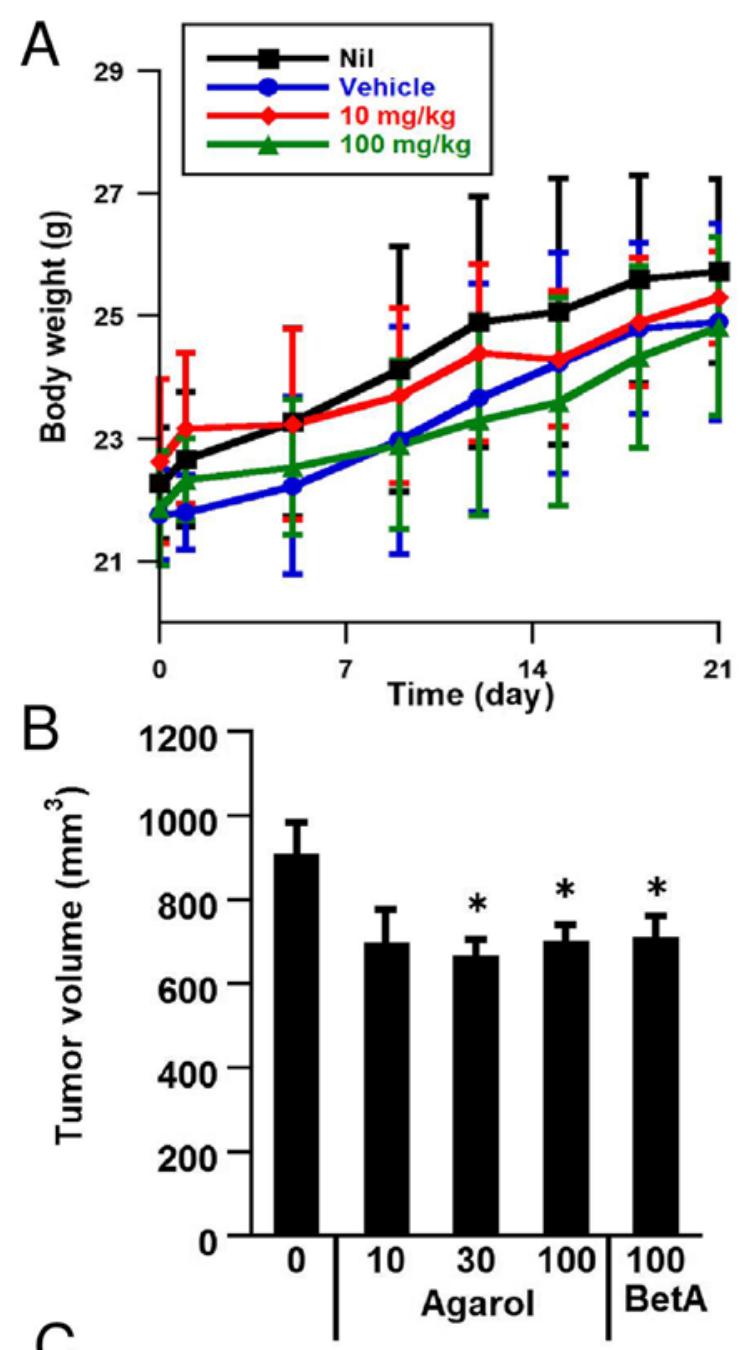

C
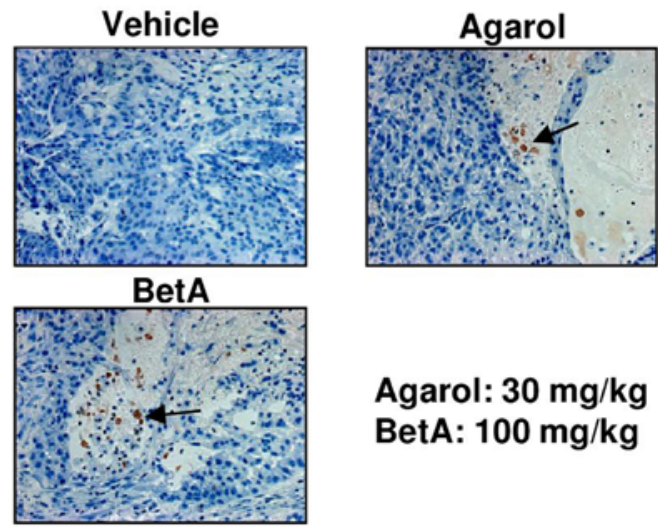

Agarol: $30 \mathrm{mg} / \mathbf{k g}$ BetA: $100 \mathrm{mg} / \mathrm{kg}$

Figure 7. Inhibition of the growth of A549 cells by Agarol in xenograft mouse model. (A) For toxicity test, respective doses of Agarol were injected into C57BL/6 mice. Total mouse body weight was recorded for 3 weeks. (B) A549 cells were subcutaneously injected into SCID mice. At week 2, mice were randomly assigned into groups: one group was i.p. injected without Agarol (control), and the other groups were injected with Agarol every 3 days for 3 weeks. Tumor volumes were measured. ${ }^{*} \mathrm{P}<0.05$. (C) The apoptotic cells in each treatment were determined in xenografts of the mice by the TUNEL staining assay. TUNEL-positive cells, with brown staining (arrow) of nucleus, in each section are indicated.

of mitochondrial ROS, further contributing to apoptotic death (21). Agarol seems to increase the activation of the apoptotic pathway in cancer cells by reducing the mitochondrial transmembrane potential with the concomitant release of ROS.
Intracellular production of ROS may activate and modulate apoptosis by regulation of p53 activity and accelerate mitochondrial depolarization during the effector phase of apoptosis (22). In turn, activation of p53 induces the production of ROS by activation or repression of genes that regulate production of ROS during apoptosis (23). Apoptosis induced by watersoluble proteoglycan is associated with the mitochondrial pathway, and is mediated by ROS generation and prolonged JNK activation (24). BetA is known to be a mitochondriotoxic drug and also induces ROS in RKO colon cancer cells (25). MKN45 cells seem to be able to activate distinct apoptotic pathways without dependence on ROS release as an apoptotic signaling amplification mechanism (data not shown).

Although caspase-dependent apoptosis is the main pathway of cell death, there is considerable evidence suggesting that caspase-independent pathways are also important. It has previously been shown that A. blazei polysaccharides effectively induce apoptosis in HL-60 cells via a mitochondria-caspase-3-dependent signaling cascade (26). An A. blazei extract by Jin et al (17), comprised of proteoglycans with a ratio (74:26) of polysaccharides to peptides, induced apoptosis in U937 cells via a caspase-3-dependent pathway. Mitochondria contain several potentially apoptogenic factors, including cytochrome $c$, procaspases-2, -3 and -9, AIF and Endo G, all of which have crucial roles in caspase-dependent or caspaseindependent apoptosis $(8,9)$. In particular, translocation of AIF, a mitochondrial flavoprotein which normally resides in the inner mitochondrial membrane, to the cytosol and nucleus results in caspase-independent apoptosis in a number of model systems (10). Importantly, various pro-apoptotic effects of AIF are not inhibited by pharmacological caspase inhibitors such as z-VAD-fmk, indicating that AIF is able to induce apoptosis in a caspase-independent manner (10). We believe that Agarol is similarly capable of inducing the translocation of AIF into the nucleus during progression of apoptosis in a caspaseindependent manner, due to the failures of the pan-caspase inhibitor to attenuate Agarol-induced AIF translocation and to rescue the cells from Agarol-mediated cell death. Several reports indicate that a generation of ROS is necessary for AIF release from the mitochondria $(27,28)$. Blazeispirol A has been shown to induce caspase-independent cell death, because it is not blocked by z-VAD-fmk, and AIF is translocated from the mitochondria to the cytosol after treatment (5).

The oral administration of A. blazei Murrill does not prevent tumor growth in SCID mice inoculated with HT-29 human colon cancer cells; however, compared with the control group, these mice showed a dose-dependent reduction in tumor growth (29). Wu et al also evaluated the A. blazei Murrill extract-dependent reduction of hepatoma formation by Smmu 7721 cells in SCID mice and metastasis formation by $\mathrm{B} 16 \mathrm{~F} 10$ melanoma cells in C57BL/6 mice (29). In vivo assays suggest a role for $A$. blazei-derived $\beta$-glucan in the initial steps of the metastatic process or intravasation in lung cancer (16). In addition, $\beta$-glucan reduced tumor burden associated with peritoneally disseminated metastasis from ovarian cancer (16). Several studies have demonstrated that various mushroom species can enhance immunity (so-called immunomodulators) through activation of natural killer cells and modulation of lymphocyte number and activity $(30,31)$. In contrast, it has been demonstrated that the purified components of 
A. blazei Murill primarily consists of $\beta$-glucan, and sensitizes doxorubicin-mediated apoptotic signaling by enhancing the accumulation of intracellular doxorubicin via the inhibition of NF- $\kappa \mathrm{B}$ activity (32). Therefore, $\beta$-glucan, when combined with low doses of doxorubicin, has the potential to provide more efficient therapeutic effects against drug-resistant human hepatocellular carcinoma (32). In xenograft mono-therapeutic experiments, there was still not a clear trend and it is probable that the animal protocol used was not sufficient to detect significant effects of Agarol. Careful animal studies are still necessary to determine whether Agarol can provide similar antitumor activities.

In conclusion, while the underlying mechanisms and actions of the newly identified Agarol remain to be elucidated, mitochondria-mediated downstream molecular events, including AIF release without activated caspase-3 expression during induction of apoptosis, as well as other related mechanisms, should be further investigated. With additional insight into the mechanisms of these compounds derived from A. blazei, current findings suggest that Agarol could be a promising modality for solid cancers.

\section{Acknowledgements}

We thank Mr. D. Mrozek for editing the manuscript.

\section{References}

1. Xu T, Beelman RB and Lambert JD: The cancer preventive effects of edible mushrooms. Anticancer Agents Med Chem 12: $1255-1263,2012$

2. Kidd PM: The use of mushroom glucans and proteoglycans in cancer treatment. Altern Med Rev 5: 4-27, 2000.

3. Ohno N, Furukawa M, Miura NN, Adachi Y, Motoi M and Yadomae T: Antitumor beta glucan from the cultured fruit body of Agaricus blazei. Biol Pharm Bull 24: 820-828, 2001.

4. Takaku T, Kimura Y and Okuda H: Isolation of an antitumor compound from Agaricus blazei Murill and its mechanism of action. J Nutr 131: 1409-1413, 2001.

5. Su Z-Y, Tung Y-C, Hwang LS and Sheen L-Y: Blazeispirol A from Agaricus blazei fermentation product induces cell death in human hepatoma Hep 3B cells through caspase-dependent and caspase-independent pathways. J Agric Food Chem 59: 5109-5116, 2011.

6. Ziliotto L, Pinheiro F, Barbisan LF and Rodrigues MAM: Screening for in vitro and in vivo antitumor activities of the mushroom Agaricus blazei. Nutr Cancer 61: 245-250, 2009.

7. Suzuki M, Endo M, Shinohara F, Echigo S and Rikiishi H: Differential apoptotic response of human cancer cells to organoselenium compounds. Cancer Chemother Pharmacol 66 : $475-484,2010$.

8. Kroemer G and Martin SJ: Caspase-independent cell death. Nat Med 11: 725-730, 2005.

9. Hirose T and Horvitz HR: An Sp1 transcription factor coordinates caspase-dependent and -independent apoptotic pathways. Nature 500: 354-358, 2013

10. Kim E-A, Jang J-H, Lee Y-H, Sung EG, Song IH, Kim JY, Kim S, Sohn HY and Lee TJ: Dioscin induces caspase-independent apoptosis through activation of apoptosis-inducing factor in breast cancer cells. Apoptosis 19: 1165-1175, 2014.

11. Yogeeswari P and Sriram D: Betulinic acid and its derivatives: A review on their biological properties. Curr Med Chem 12: 657-666, 2005.

12. Liu Y and Luo W: Betulinic acid induces Bax/Bak-independent cytochrome $\mathrm{c}$ release in human nasopharyngeal carcinoma cells. Mol Cells 33: 517-524, 2012

13. Hetland G, Johnson E, Lyberg T and Kvalheim G: The mushroom Agaricus blazei murill elicits medicinal effects on tumor, infection, allergy, and inflammation through its modulation of innate immunity and amelioration of Th1/Th2 imbalance and inflammation. Adv Pharmacol Sci 2011: 157015, 2011.
14. Ooi VE and Liu F: Immunomodulation and anti-cancer activity of polysaccharide-protein complexes. Curr Med Chem 7: 715-729, 2000.

15. Yu C-H, Kan S-F, Shu C-H, Lu T-J, Sun-Hwang L and Wang PS: Inhibitory mechanisms of Agaricus blazei Murill on the growth of prostate cancer in vitro and in vivo. J Nutr Biochem 20: 753-764, 2009.

16. Kobayashi H, Yoshida R, Kanada Y, Fukuda Y, Yagyu T, Inagaki K, Kondo T, Kurita N, Suzuki M, Kanayama N, et al: Suppressing effects of daily oral supplementation of beta-glucan extracted from Agaricus blazei Murill on spontaneous and peritoneal disseminated metastasis in mouse model. J Cancer Res Clin Oncol 131: 527-538, 2005.

17. Jin C-Y, Choi YH, Moon D-O, Park C, Park YM, Jeong SC, Heo MS, Lee TH, Lee JD and Kim GY: Induction of G2/M arrest and apoptosis in human gastric epithelial AGS cells by aqueous extract of Agaricus blazei. Oncol Rep 16: 1349-1355, 2006.

18. Rieber M and Rieber MS: Signalling responses linked to betulinic acid-induced apoptosis are antagonized by MEK inhibitor U0126 in adherent or 3D spheroid melanoma irrespective of p53 status. Int J Cancer 118: 1135-1143, 2006.

19. Green DR and Kroemer G: Cytoplasmic functions of the tumour suppressor p53. Nature 458: 1127-1130, 2009.

20. Tung Y-C, Su Z-Y, Kuo M-L and Sheen L-Y: Ethanolic extract of Agaricus blazei fermentation product inhibits the growth and invasion of human hepatoma HA22T/VGH and SK-Hep-1 cells. J Tradit Complement Med 2: 145-153, 2012.

21. Hajnóczky G, Davies E and Madesh M: Calcium signaling and apoptosis. Biochem Biophys Res Commun 304: 445-454, 2003.

22. Holley AK, Dhar SK and St Clair DK: Manganese superoxide dismutase versus p53: The mitochondrial center. Ann NY Acad Sci 1201: 72-78, 2010.

23. Maillet A and Pervaiz S: Redox regulation of $\mathrm{p} 53$, redox effectors regulated by p53: A subtle balance. Antioxid Redox Signal 16: 1285-1294, 2012.

24. Kim M-O, Moon D-O, Jung JM, Lee WS, Choi YH and Kim G-Y: Agaricus blazei extract induces apoptosis through ROS-dependent JNK activation involving the mitochondrial pathway and suppression of constitutive NF- $\kappa$ B in THP-1 cells. Evid Based Complement Alternat Med 2011: 838172, 2011.

25. Chintharlapalli S, Papineni S, Lei P, Pathi S and Safe S: Betulinic acid inhibits colon cancer cell and tumor growth and induces proteasome-dependent and -independent downregulation of specificity proteins $(\mathrm{Sp})$ transcription factors. BMC Cancer 11: 371,2011 .

26. Li X, Zhao X, Wang H, Han J and Liu L: A polysaccharide from the fruiting bodies of Agaricus blazei Murill induces caspasedependent apoptosis in human leukemia HL-60 cells. Tumour Biol 35: 8963-8968, 2014.

27. Xie J, Xu Y, Huang X, Chen Y,Fu J, Xi M and Wang L: Berberineinduced apoptosis in human breast cancer cells is mediated by reactive oxygen species generation and mitochondrial-related apoptotic pathway. Tumour Biol 36: 1279-1288, 2015.

28. Su J, Cheng H, Zhang D, Wang M, Xie C, Hu Y, Chang HC and Li Q: Synergistic effects of 5-fluorouracil and gambogenic acid on A549 cells: Activation of cell death caused by apoptotic and necroptotic mechanisms via the ROS-mitochondria pathway. Biol Pharm Bull 37: 1259-1268, 2014.

29. Wu M-F, Chen Y-L, Lee M-H, Shih YL, Hsu YM, Tang MC, Lu HF, Tang NY, Yang ST, Chueh FS, et al: Effect of Agaricus blazei Murrill extract on HT-29 human colon cancer cells in SCID mice in vivo. In Vivo 25: 673-677, 2011.

30. Wasser SP: Medicinal mushrooms as a source of antitumor and immunomodulating polysaccharides. Appl Microbiol Biotechnol 60: 258-274, 2002.

31. Nozaki H, Itonori S, Sugita M, Nakamura K, Ohba K, Suzuki A and Kushi Y: Mushroom acidic glycosphingolipid induction of cytokine secretion from murine T cells and proliferation of NK1.1 $\alpha / \beta$ TCR-double positive cells in vitro. Biochem Biophys Res Commun 373: 435-439, 2008.

32. Lee JS and Hong EK: Agaricus blazei Murill enhances doxorubicin-induced apoptosis in human hepatocellular carcinoma cells by NFкB-mediated increase of intracellular doxorubicin accumulation. Int J Oncol 38: 401-408, 2011. 